\title{
Rursus
}

m Ruksus

Poiétique, réception et réécriture des textes antiques

$1 \mid 2006$
Le modèle animal (I)

\section{Les animaux techniciens}

Réflexions sur l'animal faber vu par les Anciens

Jean Bouffartigue

\section{OpenEdition}

Journals

Édition électronique

URL : http://journals.openedition.org/rursus/48

DOI : $10.4000 /$ rursus.48

ISSN : 1951-669X

Éditeur

Université Nice-Sophia Antipolis

Référence électronique

Jean Bouffartigue, "Les animaux techniciens », Rursus [En ligne], 1 | 2006, mis en ligne le 09 juillet 2006, consulté le 10 décembre 2020. URL : http://journals.openedition.org/rursus/48 ; DOI : https:// doi.org/10.4000/rursus. 48

Ce document a été généré automatiquement le 10 décembre 2020.

Rursus 


\title{
Les animaux techniciens
}

\author{
Réflexions sur l'animal faber vu par les Anciens
}

Jean Bouffartigue

1 Tous les critères ou presque que les Anciens ont retenus pour différencier l'homme de l'animal ont été contestés par les Anciens eux-mêmes au moyen de contre-exemples pris dans le règne animal et censés prouver que les animaux disposaient de ces facultés prétendument distinctives. Ainsi en fut-il de la raison ou du langage articulé. Pour ce qui est de la faculté ou des facultés qui permettent à l'homme de fabriquer des objets, elles se voyaient opposer le cas de certains animaux dont les productions rivalisent de finesse et de fonctionnalité avec les œuvres des hommes, et même les surpassent.

2 Ces cas, il est vrai, étaient peu nombreux. Ce sont toujours les mêmes espèces qui sont citées par les Anciens quand il s'agit de montrer que l'animal peut être technicien ou artisan. Le corpus que j'utiliserai pour étudier ce thème sera composé de nos principaux informateurs grecs sur le règne animal: Aristote, Philon d'Alexandrie, Plutarque et Elien ${ }^{1}$.

3 Les animaux artisans régulièrement évoqués sont l'araignée, la fourmi, l'hirondelle, l'abeille et l'alcyon.

Philon, Alexander 17-18 (traduction A. Terian). « Prenant une matière non travaillée comme si c'était de la laine, l'araignée la façonne d'une manière très habile et artistique. D'abord elle la file très finement, comme avec la main. Ensuite, en l'étendant et l'entrelaçant, elle file et tisse avec une si merveilleuse habileté qu'elle est capable de produire une véritable œuvre d'art [...]. Il existe une autre merveille chez l'araignée, que les couturiers sont incapables d'imiter parce qu'ils divisent leurs techniques : ceux qui ont charge de filer ne tissent pas, et ceux qui tissent ne filent pas. De plus l'araignée possède en elle-même ce dont elle a besoin. "

Elien, La personnalité des animaux VI, 43 (traduction A. Zucker). «Les historiens célèbrent les galeries souterraines des Égyptiens, et ils célèbrent aussi, de concert avec la famille des poètes, certains labyrinthes crétois. Mais ils n'ont pas encore pris connaissance de la complexité des sentiers, lacets et circuits que les fourmis creusent dans la terre. [...] Quant à la terre qu'elles remontent en surface, elles en font des sortes de murs et de protections, en la disposant tout autour de l'entrée de façon à empêcher que les infiltrations d'eau de pluies ne les inondent trop facilement [...]. Elles construisent avec une grande habileté des murs de séparation entre les cavités qui permettent de les isoler les unes des autres. » 
Aristote, Histoire des animaux IX, 7, 612b 21 (traduction P. Louis). "Prenons par exemple, pour commencer, chez les oiseaux, la construction du nid de l'hirondelle. Celle-ci, avec son mortier de paille et de boue, fait ce que fait l'homme. En effet elle entremêle de la boue aux menues brindilles. Et si elle manque de boue, elle se mouille, puis elle roule ses ailes dans la poussière. De plus elle se fait un lit de paille (stibadopoiei) comme les humains, en disposant (hupotitheisa) une première couche dure et en en façonnant les contours exactement à sa taille. »

Plutarque, L'intelligence des animaux 10,966de. «Et nous avons sous les yeux les préparatifs des hirondelles avant la procréation : avec soin, elles prennent d'abord des brindilles solides pour les placer à la base, comme des fondations, puis elles modèlent un volume arrondi (periplattein) avec des éléments plus légers; et si elles s'aperçoivent que le nid a besoin de quelque enduit collant, elles vont voler au ras d'un étang, ou de la mer, effleurant de leurs plumes la surface de l'eau juste assez pour que l'humidité les mouille sans les alourdir, puis elles ramassent de la poussière et c'est ainsi qu'elles replâtrent (exaleiphein) et ressoudent (sundein) les lézardes et les éboulements. Par sa forme, l'ouvrage ne comporte ni arêtes ni faces, mais elles lui donnent, autant que possible, une surface unie et une forme à peu près sphérique : une telle construction, en outre stable et spacieuse, n'offre guère de prise aux bêtes qui voudraient l'attaquer de l'extérieur. »

Plutarque, L'intelligence des animaux 35, 983bc. «L' amour maternel de l'alcyonne et le souci qu'elle a de la sauvegarde de sa progéniture l'incitent, quand elle se rend compte qu'elle est pleine, à s'attaquer sans délai à la construction du nid. Mais elle ne malaxe (phurein) pas de la boue pour l'appliquer (prosereidein) aux murs et aux toits comme font les hirondelles; elle ne met pas en œuvre différentes parties de son corps, comme l'abeille qui utilise la masse de son corps pour pénétrer et creuser la cire tandis que ses pattes brossent (psauein) la masse compacte pour la diviser en cases hexagonales; non, l'alcyonne n'a qu'un seul et unique instrument, une seule arme, un seul outil, son bec; sans rien employer d'autre au service de son naturel laborieux et ingénieux, elle réalise une invention (mêkhanasthai) et une fabrication ( dêmiourgein) qu'on aura peine à croire possibles si l'on a pas de ses yeux observé l'ouvrage qu'elle construit, ou plutôt le vaisseau qu'elle carène (naupêgein), de tant de modèles le seul inchavirable et insubmersible. Elle recueille en effet des arêtes d'orphie, puis les réunit (suntithenai) et les attache ensemble (sundein) en les entrelaçant (enkataplekein), disposant les unes en long et les autres en travers, comme si elle introduisait une trame sur une chaîne. Elle ménage en outre, dans leur position réciproque, une courbure et un arrondi, de façon à obtenir par ce système un ouvrage rond et, de par le caractère quelque peu oblong de sa structure, assez semblable à une nasse de pêcheur. Lorsqu'elle a terminé, elle va le déposer à la limite du ressac, à l'endroit où la mer bat mollement. Si l'ouvrage est mal joint, l'action du flot lui apprend qu'elle doit le réparer et le resserrer (katapuknoun) car elle le voit se désunir sous le choc. S'il est bien assemblé, le flot le comprime et le soude, à tel point que la pierre et le fer ont du mal à le disjoindre et à l'entamer. Mais il n'est rien de plus admirable que les proportions et la forme de la cavité interne de cet objet creux, car elle est faite de telle manière que seule son ouvrière peut y trouver place; pour les autres elle est de toute part dépourvue d'entrée et entièrement secrète, de sorte que rien ne peut y pénétrer, pas même une goutte d'eau de mer. Je pense qu'il n'est personne parmi vous qui n'ait eu l'occasion de contempler ce nid.»

4 Ce dernier animal, l'alcyon, est censé être un oiseau marin, mais l'espèce n'a pu être identifiée, et aucune des espèce connues ne correspond aux indications fournies par les divers auteurs ${ }^{2}$.

5 À la liste d'animaux évoqués ci-dessus s'ajoutent parfois la perdrix, qui, d'après Elien, construit son nid selon une technique précise ${ }^{3}$, et les termites ${ }^{4}$. En revanche, le castor 
n'est connu des Anciens que pour le prix que les hommes attachent à ses testicules. Il n'est jamais question de ses constructions.

6 La description des réalisations de ces animaux artisans est proposée par la plupart des auteurs sur un ton émerveillé, Aristote seul conservant le sang-froid scientifique nécessaire. Ce qui mobilise l'enthousiasme de nos auteurs, c'est que le travail réalisé par ces animaux soutient la comparaison avec les travaux humains les plus soignés, si bien que la mise en rapport du travail animal avec le travail humain est une constante et qu'aucun auteur grec n'a pu étudier le travail animal sans le poser en comparaison avec le travail humain. Pour Aristote, c'est le modèle humain qui est primordial. Il va jusqu'à employer, à propos de l'hirondelle, un terme hautement problématique en disant que son travail est un mimêma de la vie humaine ${ }^{5}$. Que veut-il dire? Sûrement pas que chaque hirondelle imite les hommes qu'elle a vu travailler. Il faut entendre que la vie humaine et les techniques qui la rendent possible représentent l'étalon par rapport à quoi les activités animales doivent être mesurées. Et ce terme mimêma, comme certains de ses synonymes, est récurrent dans l'Antiquité grecque post aristotélicienne, et jusqu'à Origène : les animaux, écrit ce dernier, réalisent des mimêmata, des répliques des œuvres humaines, mais sans aucune intention d'imiter : c'est la nature ou c'est Dieu qui le veulent ainsi ${ }^{6}$. Les admirateurs des animaux inversent la relation: ils ne manquent pas de souligner que les réalisations animales sont plus parfaites que les réalisations humaines, et que les hommes les imitent, ou du moins tentent de les imiter, consciemment et volontairement ${ }^{7}$.

7 Aujourd'hui la curiosité la plus élémentaire pousserait quiconque serait informé de ces performances animales à s'enquérir de la façon dont ces animaux parviennent à les réaliser. Ce souci est modéré chez les anciens Grecs. Les animaux qu'ils décrivent accomplissent des opérations techniques diverses, dénotées par des verbes de métier: dans les textes cités ci-dessus on retient stibadopoiein, faire un lit, periplattein, modeler un volume arrondi, exaleiphein, replâtrer, phurein, malaxer, psauein, brosser, naupêgein, caréner, enkataplekein, entrelacer, katapuknoun, resserrer's. Ces actions sont l'effet d'une compétence ouvrière exprimée par des verbes de valeur plus générale comme mêkhanasthai et surtout dêmiourgein. C'est tout un lexique d'activités artisanales qui est employé dans ces textes. Or les Anciens ne paraissent pas prendre la mesure du caractère métaphorique de ces verbes. Ceux-ci désignent des actions proprement humaines, et l'on ne sait pas a priori comment un oiseau peut tisser ou malaxer. Avec le meilleur esprit scientifique, Aristote entreprend de répondre à la question du comment. Il observe l'araignée qui fait sa toile et en dégage le schéma suivant :

«Celle-ci commence sa toile en tendant un fil qui marque les contours des différents côtés; puis elle met en place la chaîne en partant du centre; et sur ces fils elle fait passer ceux qui constituent pour ainsi dire la trame; puis elle tisse les fils intermédiaires ${ }^{9} . »$

Mais ce schéma reste abstrait et ne répond que partiellement à la question : reste encore à savoir comment ces actes sont compatibles avec les anatomies animales. Quels sont les organes dont les animaux se servent pour opérer ces fabrications, déplacements, transformations? Il est utile de faire ici une brève revue des différents organes animaux mentionnés par les Anciens comme susceptibles d'être utilisés pour une activité de type humain. 
9 La pince est pour Aristote analogique de la main ${ }^{10}$. Mais la tradition antique ne nous fournit pas de témoignage sur un usage des pinces, chez les crustacés par exemple, qui soit assimilé à l'usage de la main chez l'homme.

A propos de la patte, Aristote écrit ceci :

« Parmi les quadrupèdes sanguins vivipares, les uns ont les pieds à plusieurs fentes, comme les mains et les pieds de l'homme (en effet certains ont plusieurs doigts, par exemple le lion, le chien, la panthère ${ }^{11}$."

11 Nous sommes toujours dans la même perspective d'anatomie comparée. Il est certain que la réalisation de l'extrémité des membres chez les quadrupèdes est plus proche anatomiquement de la main humaine que ne l'est la pince du crabe, mais physiologiquement elle en est presque aussi éloignée ${ }^{12}$. Le chien, nous dit donc Aristote, a des doigts comme l'homme. Or, comme l'homme, nous dit la tradition, il peut jeter des cailloux, et même déposer des cailloux avec une certaine précision :

« ...les corbeaux de Libye qui, lorsqu'ils ont besoin de boire, jettent des pierres dans le bassin de façon à le remplir et à faire monter le niveau de l'eau jusqu'à ce qu'elle soit à portée. Et puis voilà que, sur un bateau, j'ai observé un chien qui, en l'absence des matelots, jetait des cailloux dans l'huile d'une amphore entamée, et j'ai dû constater avec étonnement qu'il saisissait et comprenait le phénomène du liquide chassé hors d'un récipient par l'action des corps plus lourds prenant place sous les plus légers ${ }^{13}$.»

Ce n'est certainement pas avec ses extrémités pourvues de doigts que le chien est capable d'accomplir cette opération. C'est évidemment dans sa gueule qu'il est censé transporter les cailloux. L'observation la plus sommaire des mammifères nous montre que la gueule est utilisée à des fins variées. Il n'en est pourtant pas question dans notre tradition antique. Une des raisons en est évidente: les mammifères quadrupèdes ne produisent rien ; aucun ne mérite le statut d'artisan. Aucun non plus n'est capable, comme certains animaux, d'utiliser certaines parties de son corps comme des outils ou instruments humains. Aucun, sauf un, à supposer qu'il s'agisse bien d'un mammifère quadrupède : le martichoras, complaisamment décrit par Ctésias et à sa suite par Elien ${ }^{14}$. Cet animal tire à l'arc: l'arc est constitué par sa queue qu'il recourbe et détend, et les flèches par de redoutables piquants qui poussent sur son corps. La principale caractéristique de cet animal est néanmoins d'être imaginaire. Tout aussi étonnant, mais garanti par Aristote et attribué à un animal bien réel, est le comportement du nautile ou argonaute, qui est un petit céphalopode. Selon Aristote, le nautile transforme son corps en navire : il dispose d'une coquille qui lui sert de coque; ses tentacules étant reliés par une membrane, il étend celle-ci au vent en guise de voile, tandis que deux autres tentacules plongent dans l'eau à l'arrière et servent de gouvernail ${ }^{15}$. Cette notice a eu beaucoup de succès après Aristote chez les collectionneurs de mirabilia.

13 Sur les organes utilisés par les animaux artisans proprement dits, quelles informations les auteurs anciens fournissent-ils ? Sur ce sujet, Plutarque est admirablement clair. L'extrait de L'intelligence des animaux cité ci-dessus et concernant l'alcyonne nous apprend que cet oiseau accomplit tout son travail avec son bec, mais nous fait savoir en même temps que les abeilles y emploient leurs pattes ainsi que le volume de leur corps. Aristote confirme que les pattes sont les organes les plus actifs chez les abeilles :

«Les abeilles pour recueillir la cire grimpent vers les fleurs et se servent activement de leurs pattes de devant. Puis elles les essuient aux pattes médianes, et celles-ci dans le creux que forment leurs pattes de derrière ${ }^{16}$. " 
Une condition commune à toutes les représentations de l'animal artisan est l'absence de la main, cet organe indispensable de l'homo faber. Ce trait est d'une importance décisive, mais il est relativement peu commenté par les Anciens. Outre l'allusion fugace, que nous avons rencontrée chez Philon, à l'araignée qui tisse «comme avec une main $»^{18}$, on rencontre chez le chrétien Théodoret une intéressante mise en opposition des outils anatomiques dont disposent les animaux (griffes, sabots) avec la possession conjointe, chez l'homme, de l'intellect et de la main, fondements de l'activité artisane qui lui fournit ce que la nature ne lui a pas donné19. La question de la main chez l'être vivant en général a été posée dans l'Antiquité, comme nous le verrons, mais celle de la main ou de son substitut chez des espèces particulières ne l'a été qu'à propos de deux animaux qui ne sont pas des animaux artisans, le singe et l'éléphant. Aristote a noté que le singe était le seul animal autre que l'homme à être pourvu de mains, et même de quatre mains. Cependant, ajoute-t-il, ces mains ont une forme " plus bestiale », thêriôdesteron, que celles de l'homme ${ }^{20}$. Ici s'arrête sa réflexion. La main du singe n'a pas suscité chez les Anciens l'intérêt et la curiosité que l'on pouvait attendre. Si intérêt il y eut, il ne fut ni scientifique ni philosophique : ainsi s'est-on amusé à déguiser le singe en cocher, à lui faire tenir les rênes d'un attelage de chèvres et brandir le fouet ${ }^{21}$. Quant à l'éléphant, les Anciens considéraient que la nature lui avait donné, avec sa trompe, un organe presque aussi efficace que la main. C'est ce que note Aristote :

"L'éléphant a un nez d'une forme et d'une dimension telles qu'il peut s'en servir comme de mains. En effet il boit et mange en portant à sa bouche avec sa trompe, et il fait passer des objets au cornac qui est sur son dos. Avec sa trompe, il arrache aussi des arbres, et quand il marche dans l'eau, elle lui sert à respirer ${ }^{22}$."

16 Elien va sensiblement plus loin en affirmant qu'avec leur trompe les éléphants sont capables d'écrire ${ }^{23}$. Mais Aristote avait dit l'essentiel : si la trompe permet à l'éléphant de travailler, ce ne sont que travaux de force très élémentaires et qui, de plus, lui sont imposés et définis par ses maîtres humains. La main du singe et la trompe de l'éléphant, organes certes efficaces, ne permettent pas à ces animaux de fabriquer quoi que ce soit.

Il convient à présent d'examiner l'approche théorique que les Anciens ont développée au sujet des performances artisanales des animaux. Les Grecs ont reçu d'Aristote la première leçon à ce sujet. Sans les priver du plaisir d'admirer les performances des animaux, Aristote leur a suggéré de n'y pas voir les effets d'une intelligence artiste, mais de la nature réalisant ces finalités directement grâce aux organes des animaux.

«Mais c'est surtout visible pour les animaux autres que l'homme, qui n'agissent ni par art (tekhnêi) ni par recherche, ni par délibération; d'où cette question: les araignées, fourmis et animaux de cette sorte travaillent-ils avec l'intelligence (nôi) ou quelque chose d'approchant? [...] Si donc c'est par une impulsion naturelle ( phusei) et en vue de quelque fin que l'hirondelle fait son nid et l'araignée sa toile [... ], il est clair que cette sorte de causalité existe dans les êtres naturels ${ }^{24}$. »

18 Cette remarquable déclaration doit être cherchée en dehors des ouvrages zoologiques d'Aristote. Dans les dits ouvrages zoologiques, Aristote n'exprime pas une autre doctrine, mais il est vrai qu'il lui arrive plus d'une fois de faire appel à la notion de tekhnê à propos des animaux. Ainsi distingue-t-il parmi les «insectes » les araignées lisses et grêles qui 
sont les plus tekhnikoi ${ }^{25}$, et parmi les oiseaux nidificateurs ceux qui sont eumêkhanoi ${ }^{26}$. Même des animaux marins sont qualifiés de tekhnika : la raie torpille, la baudroie, et bien d'autres encore ${ }^{27}$. Il faut sans doute entendre que la technique déployée par ces animaux est différente de la technique humaine. Aristote révèle le fond de sa pensée dans un autre texte :

«La douceur et la sauvagerie, l'humeur facile et le mauvais caractère, le courage et la lâcheté, les craintes, les audaces, les colères, les ruses, les traits d'intelligence appliqués à la pensée constituent des ressemblances (homoiotêtes) avec l'homme qu'on retrouve chez beaucoup d'animaux [...]. Car certains animaux diffèrent de l'homme selon le plus et le moins [...]; d'autres au contraire diffèrent de lui selon un rapport d'analogie (to analogon) : ainsi à ce qui est chez l'homme technique, sagesse, intelligence correspond chez certains animaux quelque autre faculté naturelle du même genre ${ }^{28}$. »

19 Ainsi donc, ce qu'on peut appeler tekhnê chez les animaux n'est pas ce qu'on appelle de ce nom chez les hommes. Dans le cas de la tekhnê il n'y a pas entre animal et homme un rapport quantitatif, mais qualitatif. L'animal n'est pas plus ou moins technicien que l'homme. Il l'est autrement : il dispose d'une faculté analogue à la capacité technicienne, qui n'a pas reçu de nom spécifique. La capacité dont il dispose ressemble à la tekhnê, mais n'est pas de la tekhnê. Cette analogie ou ressemblance autorise cependant à user à son propos du terme tekhnê ou de ses dérivés : Aristote dans de tels domaines se dispense de tout rigorisme terminologique ${ }^{29}$. Par ailleurs il recourt à une autre catégorie classificatoire. Les animaux dont nous parlons ici sont volontiers qualifiés au moyen de l'adjectif ergatikos ${ }^{30}$. Technique ou instinct, ces animaux travaillent ${ }^{31}:$ la notion est particulièrement martelée dans la longue description de la vie des abeilles au chapitre 40 du livre IX de l'Histoire des animaux.

«Les abeilles sont spécialisées dans chaque genre de travail (hekastôn tôn ergôn) : par exemple les unes apportent le suc des fleurs, d'autres vont chercher l'eau, d'autres lissent et alignent les rayons ${ }^{32}$.»

La tranquillité avec laquelle Aristote recourt à propos des animaux à des concepts liés à la technique et au travail ne suffit pas à le rendre suspect d'anthropomorphisme. La force de la position aristotélicienne est qu'elle lève la difficulté qui surgit dès lors qu'on admet que les animaux accèdent à la production technique. On est tenté de conclure que leurs œuvres s'inscrivent dans la catégorie de l'art opposée dans une polarité bien connue à celle de la nature. Mais Aristote nous en dissuade : la toile d'araignée, pose-t-il, n'est pas une œuvre d'art, c'est un objet naturel, comme l'araignée elle-même.

21 Aristote a su également éclairer le rapport entre la capacité productive caractérisant l'homme et l'existence de la main, et même d'une main possédant un pouce opposable.

"L'homme, au lieu des pattes et des pieds de devant, possède des bras et ce qu'on appelle des mains. Car il est le seul des animaux à se tenir droit parce que sa nature et son essence sont divines. Or la fonction de l'être divin par excellence est la pensée et la sagesse. Mais cette fonction n'aurait pas été facile à remplir si la partie supérieure de son corps avait pesé lourdement. Car la pesanteur enlève toute souplesse au raisonnement et au sens commun. Aussi, quand la pesanteur et l'élément corporel l'emportent, le corps s'incline nécessairement vers la terre ${ }^{33}$. » «De même que sans main du tout il ne serait pas possible de prendre, de même on ne le pourrait si le pouce n'existait pas $^{34}$.»

Le premier des deux textes cités ici définit sans ambiguïté l'ordre des causes. L'homme est divin, donc il doit pouvoir exercer sa fonction divine, donc il ne doit pas avoir une tête lourde et penchée vers le sol, donc il se tient droit. Au contraire chez d'autres animaux le 
corporel l'emporte sur la pensée, et donc ils se tiennent penchés vers la terre. Une telle conception de l'ordre des causes amène Aristote à disqualifier l'intuition d'Anaxagore sur le rôle de la main dans l'accession de l'homme à l'intelligence.

" Anaxagore dit que c'est parce qu'il a des mains que l'homme est le plus intelligent des animaux. Il est plus rationnel de dire qu'il a des mains parce qu'il est le plus intelligent. Car la main est un outil (organon) ; or la nature attribue toujours, comme le ferait un homme sage, chaque organe (organa) à qui est capable de s'en servir ${ }^{35}$. »

Pourtant, c'est bien Anaxagore, et non Aristote, que les observations de la science moderne justifient sans relâche. Le processus d'hominisation parait bien s'être déroulé à l'envers de ce qu'asserte le Stagirite ${ }^{36}$ : d'abord la station debout, puis en conséquence la libération de la main, grâce à laquelle l'homo est devenu faber. Mais parler ici de processus d'hominisation est un anachronisme épistémologique. Ni Aristote ni Anaxagore ne l'envisagent, et tous deux se rejoignent dans l'idée d'une conjonction nécessaire et intemporelle entre l'intelligence humaine et la possession de la main; il s'agit en fait d'une implication réciproque; Anaxagore dit : s'il y a main il y a intelligence, et Aristote : s'il y a intelligence il y a main.

Savoir si la capacité technique est la cause qui engendre le développement des capacités intellectuelles est un problème complexe. Dans une zoologie "à l'antique ", on ne manquerait pas de faire apparaître que les animaux techniciens, si admirables que soient leurs réalisations, n'ont pourtant pas accédé au même niveau d'intelligence que l'homme. Dans leurs réflexions sur le rôle décisif de la main, les Anciens n'ont jamais invoqué l'acquisition des capacités techniques comme une étape dans l'accession à l'intelligence. Le processus décrit par le chrétien Grégoire de Nysse est d'un tout autre modèle : la main favorise l'intelligence parce qu'indirectement elle permet la parole.

«Si l'homme n'avait pas de main [scil. pour recueillir sa nourriture et la porter à sa bouche], son visage serait conformé de façon à servir à son alimentation, comme chez les autres quadrupèdes : il aurait un visage allongé vers l'avant, terminé par le nez, des lèvres épaisses et spécialement faites pour happer l'herbe, une langue volumineuse, raide et rugueuse [...]. Si donc il n'avait pas de mains, comment pourrait-il articuler des mots puisque sa bouche ne serait pas adaptée à l'usage de la parole $^{37}$ ?»

Comme Aristote, Grégoire de Nysse subordonne la possession des mains à une fin déterminée préalablement, à savoir l'exercice de l'intelligence ou de la raison. Comme Aristote il pense que Dieu (Aristote dirait «la nature») a équipé l'homme de la main parce qu'il (elle) l'avait fait être pensant. Il est gênant d'être en mesure d'opposer à Aristote le contre-exemple du singe. Le singe n'a pas la capacité mentale d'utiliser les ressources de la main, mais la nature lui en a tout de même donné une. Est-ce en pensant à cette difficulté qu'Aristote déclare que la main du singe est plus bestiale, ce qui en soi n'a pas grand sens ${ }^{38}$ ? Si Aristote renonçait à appliquer ici son principe selon lequel la nature ne fait rien en vain, sa position serait cohérente : l'existence de la main chez le singe, une main qui ne sert pas à être intelligent, lui permettrait de prouver qu'Anaxagore se trompe.

La position générale d'Aristote relativement aux performances artisanales des animaux, avec sa notion d'une espèce de tekhnê sans tekhnế $\hat{e}^{39}$, a eu néanmoins un impact considérable sur la pensée grecque postérieure. Non qu'elle ait dominé sans conteste, loin de là, puisque tout le courant platonicien ou presque a adhéré à l'idée que les animaux possédaient une part de raison, possession dont les performances artisanales étaient censées constituer l'un des indices. Mais elle a armé la doctrine stoïcienne de l'animal, et 
a fourni au christianisme un solide argumentaire pour proclamer que le seul partenaire de Dieu pourvu d'une âme raisonnable était l'homme. En outre elle n'a nullement désenchanté le monde. Les animaux peuvent paraitre quelquefois accomplir mieux que l'homme certains travaux caractéristiques de la vie civilisée. Les Anciens l'avaient fort bien remarqué, et leur admiration et leur émerveillement n'étaient pas diminués même lorsqu'ils refusaient aux animaux la possession de la raison. Si les travaux des animaux sans raison $(a \log a)$ sont si parfaits, c'est, pensaient-ils, parce que la grande nature y est directement à l'œuvre. Aristote est le premier à déclarer, au début des Parties des animaux, qu' i il y a beaucoup plus de finalité et de beauté (to kalon) dans les œuvres de la nature

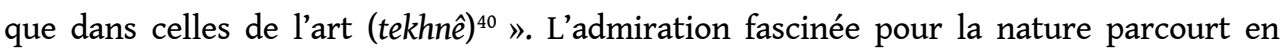
particulier l'œuvre d'Elien. Si l'on ne trouve pas chez lui de référence à cette puissance de la nature à propos des exemples qu'il donne d'animaux artisans, il est néanmoins clair que les performances techniques des animaux s'inscrivent à ses yeux dans le même ensemble qui comprend aussi les performances physiques, intellectuelles et morales. Or à de nombreuses reprises ces performances-là sont présentées comme l'effet d'une capacité naturelle, appelée phusis et qualifiée de merveilleuse (thaumastê) et de mystérieuse ( aporrêtos). Comme le dit Elien, les performances techniques de l'homme peuvent certes être admirables, mais elles n'ont rien qui passe l'entendement : elles s'expliquent parce que «l'homme est un animal doué de raison et capable de pensée et de réflexion »" Les merveilleuses réalisations des animaux artisans sont quant à elles offertes (Elien emploie souvent dans ce contexte le nom dôrea, don) par une nature généreuse et secrète. Quant au chrétien Origène, il affirme que la technique à proprement parler n'appartient qu'à l'homme, et que cette exclusivité fait partie du plan de Dieu qui a créé l'homme physiquement indigent et mal protégé dans l'intention précise de le voir développer, pour compenser ce manque, des tekhnai diverses. Quant aux réalisations animales, il invite à y voir une preuve de plus de la grandeur de Dieu, créateur de la nature qui est la cause directe de ces merveilles.

\section{NOTES}

1. S'il s'agissait de traiter notre thème dans l'ensemble de l'Antiquité, et non pas seulement dans le domaine grec, il faudrait naturellement tenir compte également de l'Histoire Naturelle de Pline. Mais, par rapport aussi bien à Aristote qu'aux autres auteurs grecs Pline ne présente que peu de singularités dans le domaine considéré.

2. Sur le nid de l'alcyon, voir en particulier Aristote, H.A. IX, 14, 616a 19-32.

3. Elien, La personnalité des animaux III, 16.

4. Ibid., XVI, 15.

5. Aristote, H.A. IX, 7, 612b 21.

6. Origène, Contre Celse IV, 81.

7. Plutarque, L'intelligence des animaux 10,966e : « les travaux de l'araignée, qui servent de modèle (arkhetupon) pour les femmes au métier et pour les chasseurs au filet ». 
8. Les verbes grecs employés par Philon dans l'Alexander ne peuvent qu'être conjecturés à partir de la traduction arménienne, la seule version qui nous reste : sont évoquées, à propos de l'araignée, les actions de façonner, filer et tisser (voir supra).

9. Aristote, H.A. IX, 39, 623a 8-11.

10. Ibid. I, 1, 486a 20.

11. Ibid. II, 1, 499b6-8.

12. Le cas du singe est particulier, et sera traité plus loin.

13. Plutarque, L'intelligence des animaux 10, $967 \mathrm{a}$.

14. Elien, La personnalité des animaux IV, 21.

15. Aristote, H.A. IX, 37, 622b 5-14.

16. Ibid. IX, 40, 624a 33 - 624b 2.

17. Elien La personnalité des animaux III, 24.

18. Philon, Alexander 17, voir supra.

19. Théodoret, De la providence IV, $612 \mathrm{~d}-613 \mathrm{~d}$.

20. Aristote, H.A. II, 8, 502, 3-4.

21. Philon, Alexander 23 ; Elien, P.A. V, 26.

22. Aristote, H.A. II, 1, 497b 26-30.

23. Elien, P.A. II, 11.

24. Aristote, Physique II, 8, 199a 21.

25. Aristote, H.A. IX, 38, 622b 23.

26. Ibid. IX, 11, 614b 34.

27. Ibid. IX, 37, 620b 10.

28. Ibid. VIII, 1, 588a 21-31.

29. Ailleurs il s'autorise à parler à propos des animaux d'une vertu comme la sôphrosunê, alors qu'il est convaincu qu'il n'y a chez les animaux ni vertu ni vice : ces mots, dit-il, doivent être entendus comme métaphoriques, kata metaphoran (Ethique à Nicomaque VII, 7, 1149b 32-34).

30. Aristote, H.A. IX, 38, $622 \mathrm{~b} 19$.

31. ergazontai, ibid. IX, 38, 622b 26.

32. Ibid. IX, 40, 625b 18-20. On note qu'Aristote n'use pas d'un dérivé de ergon pour désigner les abeilles que nous appelons « ouvrières » : il les nomme hai khrêstai melittai (624b 30).

33. Aristote, Parties des animaux 686a 24.

34. Ibid. $687 \mathrm{~b} 12$.

35. Ibid. 686b 8. Voir sur la problématique afférente à ce texte l'important article de ZUCKER Arnaud, «La main et l'esprit. Sur l'aphorisme d'Anaxagore (frg. A 102) », in GALY J.M.et GUELFUCCI M.R., éds., L'homme grec face à la nature et face à lui-même (Mél. A. Thivel), Nice 2000, p. 277-308; voir aussi, dans le même recueil, ROSELLI Amneris, « Il volto e le mani. Materiali per un commento a Greg. Nyss. De Hominis opificio, cap. 8 ", p. 310-323. 36. Grégoire de Nysse, La création de l'homme 8, PG 44, 147D-148A. Ce passage est reproduit et commenté par A. Roselli dans l'article cité note précédente.

37. Galien, quant à lui, parle, à propos du singe, d'un organe « semblable à la main » : De usu partium II, 116, 23 Heimreich.

38. Encore Aristote ne fait-il pas crédit à l'artisan humain de la sophia par cela seul qu'il possède une tekhnê : pensons au célèbre passage de Politique, 1253b 27-38, où les subordonnés, même pourvus d'une capacité technique comme les pilotes de navire, ne sont autres choses que des instruments animés employés par leurs maittres; et voir A. Zucker, op. cit. supra n. 34, p. 302. 
39. Aristote, Parties des animaux 639b 19-20.

40. Elien, P.A. II, 11.

41. Origène, Contre Celse IV, 76. L'effet positif du dénuement primitif de l'homme est un écho du mythe du Protagoras, dont la leçon a été retenue durant toute l'Antiquité, tout particulièrement par les chrétiens : voir aussi Grégoire de Nysse, La création de l'homme $141 \mathrm{~b}$; Némésios, La nature de l'homme I, 51-52, p. 9, 8-19 Morani ; Théodoret, De la providence IV, 612d - 613d, déjà évoqué supra.

\section{RÉSUMÉS}

Un petit nombre d'espèces animales, oiseaux et insectes essentiellement, sont reconnus par les Anciens capables de fabriquer des objets. La facture de ces objets est jugée admirable, et la tendance à une conception anthropomorphique du travail animal est encore favorisée par le fait que les Anciens ne s'intéressent guère au fait que l'animal, privé de main, procède d'une façon absolument différente de l'artisan humain. A leurs yeux, la possession d'une main apte au travail va de pair avec la possession de l'intelligence et de la raison (mais il peut y avoir de la raison sans main). Pour ceux qui accordent aux animaux la possession de la raison (Plutarque, et avant lui l'adversaire de Philon), leurs performances techniques sont du même type que les performances humaines. Pour ceux qui au contraire la lui refusent (Aristote, Philon, Elien), la tekhnê de l'animal, sous le même nom, désigne en réalité une faculté différente de la tekhnê humaine.

A few species of animals, mostly birds and insects, are for the Ancients admittedly able to make things. The working of those things is admired, and there is a trend to conceive the work of the animal like a man's one ; this is made stronger by the fact that the Ancients do not point out that the animal, being deprived of hand, proceeds in a way totally different from the human craftsman. They consider that possessing a hand fit for work always goes along with possessing reason. Those who allow to animals the possession of reason (like Plutarch and, before him, Philo's opponent), think that their technica performances are of the same kind as human ones. Those who deny it (Aristotle, Philo, Ælian) suppose that the tekhne of the animal, under the same name as human tekhne, in fact designates some different ability.

INDEX

Mots-clés : oiseaux, intelligence animale, technique, Aristote, anatomie comparée, Elien, Philon

\section{AUTEUR}

\section{JEAN BOUFFARTIGUE}

Université de Paris X 